However, the bonding must not be so strong as to prevent controlled movement of the molecule by the STM tip. The chemical bonds within the molecule, on the other hand, must resist being changed or broken when the molecule is pushed.

Following molecular mechanical simulations carried out at the CNRS in Toulouse, a Cu-porphyrin molecule was selected for the pushing experiments. It consisted of a stable porphyrin ring with four strongly, yet flexibly bonded, hydrocarbon groups. These groups form "legs" which are attached perpendicular to the plane of the ring owing to rotation by steric forces. Consequently, the saturated t-butyl groups that form part of the legs can dominate the molecule-surface interaction which is largely determined by van der Waals forces. A copper (100) surface was chosen because it offered the optimum adsorption characteristics of labile bonding with t-butyl groups.

Well-ordered islands of the Cu-TBPporphyrin were grown by annealing a 10$15 \%$ monolayer coverage. Individual molecules revealed themselves as a symmetric arrangement of four bright lobes (see figure). Before repositioning was started, a selected area was repeatedly imaged to ensure that significant molecular motion was not taking place. By displacing the STM tip, individual molecules were then pushed owing to tip-molecule repulsion. For instance, in the figure a rectangular cluster of six atoms was distributed by cruising through the cluster. Subsequently, each molecule was pushed to reform a cluster with an hexagonal shape that is not normally found on $\mathrm{Cu}$ (100).
The computer simulations explained why the molecule is displaced. The molecule is "squeezed" as the STM tip approaches owing to an increasing repulsive force. The molecule's legs open asymmetrically until the energy barrier for lateral displacement is overcome, at which point the molecule relaxes by taking several lateral steps via an uncorrelated slip-stick action of each of the legs. This action involves a lower energy barrier for lateral motion than for a rigid molecule. So the condition for pushing is determined by the potential barrier for lateral displacement and the molecule's internal flexibility. The mechanisms involved are characteristically different from the sliding used in lowtemperature manipulations, and understanding molecular mechanics is vital for room-temperature molecular engineering.
Stages in the construction of a hexameric ring of six CuTBP-porphyrin molecules. An as-grown rectangular array was disassembled and then each molecule was repositioned to form a hexagonal ring. Note that the three clustered molecules in the upper part of the STM images remain unchanged during the repositioning process.
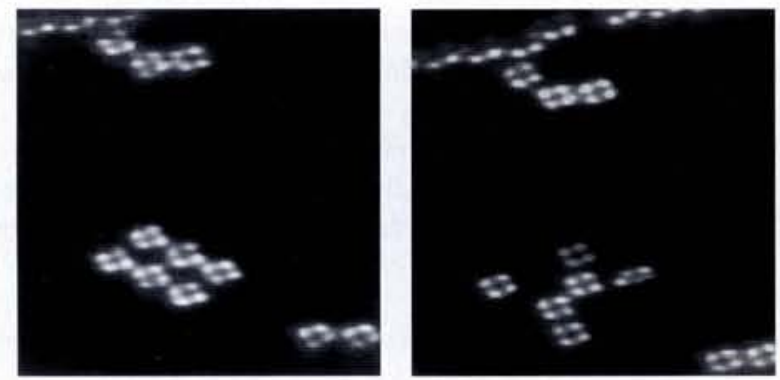

\title{
Antihdyrogen Flashes into View
}

P.A.M. Dirac proposed antimatter in 1931 and electron-positron pairs were detected soon after in cosmic rays. The CPT theorem, which can be derived from the combined operation of charge conjugation (C), space reflection $(\mathrm{P})$ and time reversal $(\mathrm{T})$ in relativistic quantum field theory, predicts that each fundamental particle has an antiparticle with the same value but opposite sign for charge and magnetic moment. As there is no reasonable doubt about the symmetry between particles and antiparticles, it should be possible to combine antiparticles into antimatter under the same forces as particles bind to form normal matter. Nine anti-hydrogen antiatoms were detected by a time-of-flight technique last September and October during an elegant experiment lasting 15 hours using collisions between antiprotons and xenon clusters at CERN's LEAR ring.

CERN hoped to make an announcement to Council in December but following normal policy waited until the Collaboration's publication (received on 8 December) was accepted by Phys Lett B for publication (in a revised form) on 21 December. The preprint "Production of Antihydrogen" was available on the CERN preprint server on 4 January, the same day as the announcement.

For most physicists, the report represents a step of the road to a new type of experiment designed to test fundamental physical principles, because antihydrogen is well-suited for $\mathrm{CPT}$ violation studies under different forces. The challenge will be to make extremely precise spectroscopic measurements of the energy levels on relatively large quantities of antihydrogen produced by combining antiprotons and positrons stored in magnetic traps. This seems feasible, but is several years away. Nonetheless, CERN's antihydrogen announcement generated enormous interest world wide, and made headlines everywhere, stirred up in part an interview published by the French newspaper Libération of Walter Oelert (Forschungszentrum Jülich), the head of the Collaboration, which discussed bomb applications of the energy released when matter and antimatter annihilate. In fact, only a few media took up this theme (e.g., The Financial Times). Apart from the inevitable references to the "fuel of science fiction" for spacecraft (The Times, London; Der Spiegel), most discussed antimatter's relevance to the debate on $L e$ Monde, New York Herald Tribune) or took a neutral view ("first step in the antiperiodic table" - New Scientist; "opening the door to a systematic study of the antiworld" - Journal de Genève).

The main problem is that antihydrogen work, as well as hyperfine spectroscopy on "atomcules" in which a helium atom's electron is replaced by a positron [see EN 26 (1995) 57], is carried out at the veteran LEAR facility which shuts down at the end of 1996. An antihydrogen experiment at Fermilab in the US has been delayed and will now be completed next year. Following a 1995 feasibility study of a simplified low-energy antiproton source called the Antiproton Decelerator (AD) based on using the accumulator part of the LEAR facility, CERN has indicated that it may be prepared to support the AD provided some 7 M\$US can be found. The next milestone will be a scheduled meeting of CERN's SPS-LEAR Experiments Committee in March (two letters-of-intent for antimatter experiments have already been announced). As the US does not have a similar project on the table, attention is also focussed on the Japan Hadron Project - a multidisciplinary facility at KEK, Tsukuba, based on a $50 \mathrm{keV}$ high-intensity proton accelerator with a beamline for antiproton experiments. A detailed JHP budget request is being prepared for submission in 1997 and completion within five years of full approval is envisaged. Meanwhile, Japanese groups playing a leading role in CERN's atomcule experiments may be interested in participating in the $\mathrm{AD}$. 